\title{
Direct imaging of quantum wires nucleated at diatomic steps
}

\author{
S. I. Molina ${ }^{a)}$ and M. Varela \\ Materials Science and Technology Division, Oak Ridge National Laboratory, Oak Ridge, \\ Tennessee 37831, USA \\ D. L. Sales and T. Ben \\ Departamento de Ciencia de los Materiales e I.M. y Q.I., Facultad de Ciencias, Universidad de Cádiz, \\ Campus Río San Pedro, Puerto Real, 11510 Cádiz, Spain \\ J. Pizarro and P. L. Galindo \\ Departamento de Lenguajes y Sistemas Informáticos, CASEM, Universidad de Cádiz, \\ Campus Río San Pedro, Puerto Real, 11510 Cádiz, Spain \\ D. Fuster, Y. González, and L. González \\ Instituto de Microelectrónica de Madrid (CNM, CSIC), Isaac Newton 8, Tres Cantos, 28760 Madrid, Spain
}

\author{
S. J. Pennycook \\ Materials Science and Technology Division, Oak Ridge National Laboratory, Oak Ridge, \\ Tennesse 37831, USA
}

(Received 8 June 2007; accepted 7 September 2007; published online 2 October 2007)

\begin{abstract}
Atomic steps at growth surfaces are important heterogeneous sources for nucleation of epitaxial nano-objects. In the presence of misfit strain, we show that the nucleation process takes place preferentially at the upper terrace of the step as a result of the local stress relaxation. Evidence for strain-induced nucleation comes from the direct observation by postgrowth, atomic resolution, $Z$-contrast imaging of an InAs-rich region in a nanowire located on the upper terrace surface of an interfacial diatomic step. (C) 2007 American Institute of Physics. [DOI: 10.1063/1.2790483]
\end{abstract}

Atomic steps located at the surfaces of substrates play a central role in controlling the growth mechanism of a wide range of materials. The effect of such steps on the growth process, the morphology, the stress and strain distributions, and the functionality of the materials has been extensively investigated. ${ }^{1-11}$ In particular, these steps are thought to constitute a heterogeneous source of nucleation for the formation of nano-objects and structural defects at the initial stages of the growth of many semiconductor nanostructures. ${ }^{12,13} \mathrm{It}$ is critically important to identify the nucleation sources for individual nano-objects such as quantum dots and wires because they constitute the fundamental blocks of future nanoelectronics and nanophotonics devices. ${ }^{14}$ The ability to control the nucleation of these nano-objects will contribute significantly to the development of reliable single-photon sources for applications in quantum information technology. ${ }^{15-17}$

Nanowires constitute a special case of self-assembled nano-objects. ${ }^{18}$ Self-assembled nano-objects can be formed spontaneously via the Stranski-Krastanov growth mode for certain semiconductor materials when a few monolayers are epitaxially deposited on a lattice-mismatched substrate. ${ }^{19,20}$ Strain is widely accepted to be the driving force for the nucleation of these nano-objects, but as yet there has been no direct experimental evidence for the role of steps, and their associated stress enhancement, in the self-organized growth of nanowire arrays. ${ }^{21,22}$

In this letter we show the direct imaging, by aberrationcorrected Z-contrast scanning transmission electron micros-

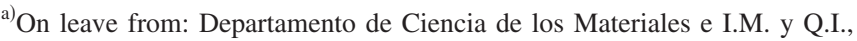
Facultad de Ciencias, Universidad de Cádiz, Campus Río San Pedro, Puerto Real, 11510 Cádiz, Spain. Electronic mail: sergio.molina@uca.es and molina@ornl.gov
}

copy (STEM) and atomic force microscopy, that, in the presence of misfit strain, nanowires preferentially nucleate on the upper terrace of a diatomic step. With finite element elasticity calculations, we demonstrate that the driving force is the stress relaxation at the upper terrace of a diatomic step, which explains the observed nucleation site of semiconductor nanowires.

The nanowires investigated consist of $\operatorname{InAs}(\mathrm{P})$ selfassembled quantum wires grown by solid source molecular beam epitaxy (MBE) on InP (001) substrates. The lattice mismatch between InAs and InP is $3.2 \%$. The control of the relaxation process of these nanostructures has recently been followed with high precision by in situ accumulated stress measurements from the initial phases of the self-assembly process. $^{23}$ The process of formation of these wires is controlled in such a way that it is possible to intentionally modify their dimensions and density with a high level of accuracy and reproducibility. These characteristics make them ideal nano-objects for detailed study, at the atomic level, of their nucleation process.

References 23 and 24 explain in detail the growth procedure for the samples (A and B) studied here. In sample A, after growing a 180-nm-thick InP buffer layer, the InP surface was exposed to $\mathrm{As}_{4}$ flux for $3 \mathrm{~s}$ at a substrate temperature $T_{S}=480^{\circ} \mathrm{C}$. A 1.5 monolayer of InAs were deposited at a deposition rate of 0.5 monolayer per second $\left(\mathrm{ML} \mathrm{s}^{-1}\right)$ and $T_{S}=480{ }^{\circ} \mathrm{C}$. After InAs deposition, the surface was annealed for 1.5 min under $\mathrm{As}_{4}$ flux. In sample $\mathrm{B}$, quantum wires were formed by depositing $1.7 \mathrm{ML}$ of InAs at $0.1 \mathrm{ML} / \mathrm{s}$ and $515^{\circ} \mathrm{C}$. The quantum wires grown using these conditions, which are completely formed well beyond the initial stages, were covered by a 20 -nm-thick InP cap layer grown by atomic layer $\mathrm{MBE}$ at $380{ }^{\circ} \mathrm{C}$. Quantum wires of sample A correspond to the initial stage of their formation process and 


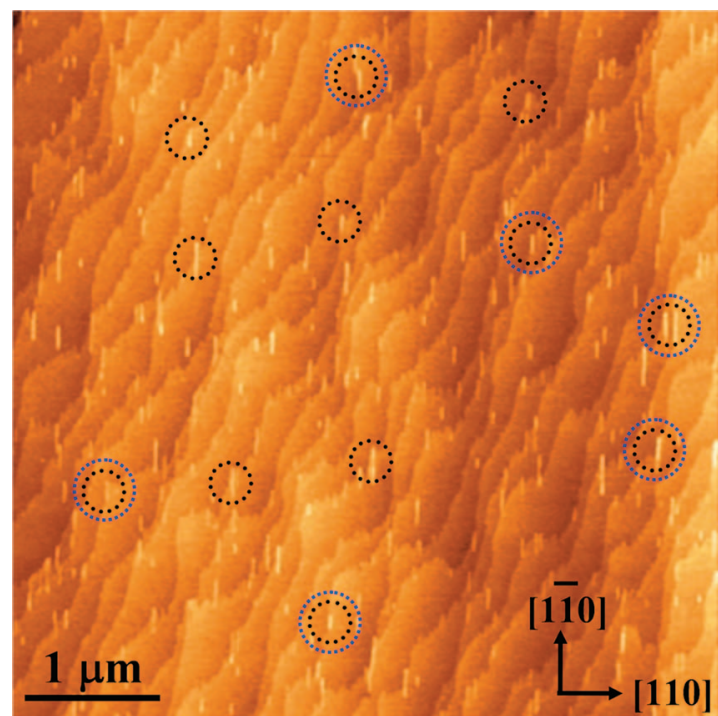

FIG. 1. (Color) AFM image of the growth surface during the initial stage of nanowires formation. Note that most of nanowires are located on the upper terraces close to the step boundary. Similarly nucleated nanowires are highlighted on the AFM image with dotted circles. The nanowires circled in blue are seen to run parallel to the step edge for practically their entire length whereas most nanowires cross the step at a small angle.

were studied by atomic force microscopy (AFM), while in contrast, fully formed wires in sample B were used for the $Z$-contrast investigations.

Figure 1 shows a representative AFM image of the growth surface during the initial stage of formation of the nanowires of sample $\mathrm{A}^{23}$ The nanowires are oriented along $[1 \overline{1} 0]$ and mostly located on the upper terraces, close to the boundaries between the upper and lower terraces of a step. The majority of the pixels associated with nanowires in the AFM image (76.8\%) are located on the upper terraces close to the step edge. In most cases, the nanowires run at a shallow angle to the step. When imaged in cross section, these nanowires would not show any clearly defined step at the substrate/wire interface. However, a small fraction of nanowires, circled in blue, run parallel to the step for the majority of their length. In these cases, the step would be parallel to the beam direction for the entire thickness of a cross section specimen.

Anisotropic elastic theory has been applied to determine by the finite element method the distribution of strain and stress generated on the upper and lower terraces of a diatomic step during the very initial stage of InAs MBE deposition. The upper terrace surface exhibits a large positive strain relative to the lower terrace from the very initial stage of the nano-object growth, even when only one InAs monolayer has been deposited. After capping the InP diatomic step with two or more InAs monolayers, the strain along [1 $1 \overline{1} 0]$ is positive on the upper terrace and is negative on the lower terrace. For all cases considered, the strain values at the upper terrace surface become larger near the edge of the step. These general findings hold also for wetting layers of $\operatorname{InAs}_{x} \mathrm{P}_{1-x}$ (instead of pure InAs) and even for situations where the surface diatomic step is displaced with respect to its original position at the InP surface during the growth of the first InAs monolayers.

The large positive strains at the upper terrace close to the step edge will act to lower the chemical potential for InAs growth at that location of the upper terrace. The chemical Downloaded 31 Mar 2009 to 161.111.235.57. Redistribution subject

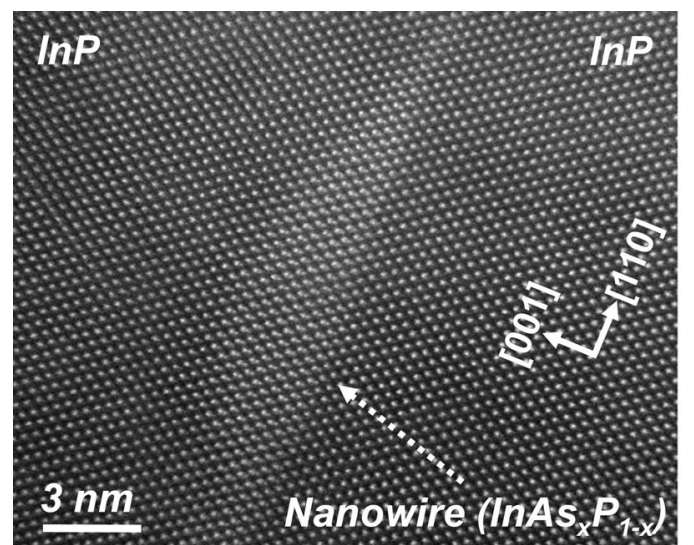

FIG. 2. Z-contrast image (raw data) of an InP capped $\operatorname{InAs}_{x} \mathrm{P}_{1-x}$ nanowire grown by solid source molecular beam epitaxy on (001) InP substrate. This image, taken with a dedicated aberration-corrected STEM VG HB603, enables the two fcc sublattices of In (brighter contrasts) and $\mathrm{As}_{x} \mathrm{P}_{1-x}$ atoms constituting the zinc-blende structure of the $\operatorname{InAs}_{x} \mathrm{P}_{1-x}$ disordered alloy to be directly distinguished. Stronger intensities are observed in the projected positions of the $\mathrm{As}_{x} \mathrm{P}_{1-x}$ atomic columns.

potential is reduced compared to a thin InAs layer pseudomorphically grown on a flat InP substrate. This result is in complete accord with the AFM observations and leads us to propose that the preferential nucleation on the upper terrace surface initiates the two-dimensional-three-dimensional transition, that is, the appearance of a nanowire seed in this preferential location triggers the Stranski-Krastanov growth transition. We expect this will apply in general to any nanoobject having a lattice mismatch with respect to the substrate material. ${ }^{25,26}$

This strain-driven mechanism would be in addition to any Ehrlich-Schwoebel step-edge barrier. However, it has been shown that such a barrier is not necessary to account for roughening on a polar semiconductor surface that undergoes a deep surface reconstruction, ${ }^{27}$ such as the $\mathrm{In}_{x} \mathrm{As}_{1-x} \mathrm{P}$ studied here. Our results demonstrate that the nucleation of nanowires on the upper terraces of the surface steps can be fully

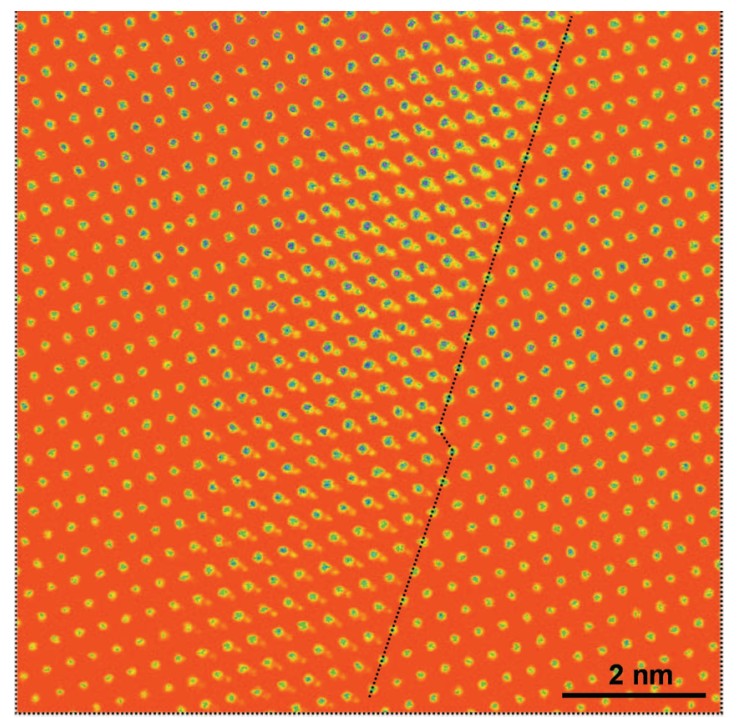

FIG. 3. (Color) Low pass filtered high resolution $Z$-contrast image obtained from a magnified region of the central part of the $\operatorname{InAs}_{x} \mathrm{P}_{1-x} / \mathrm{InP}$ nanowire interface. The existence of a diatomic step is clearly visible on the dotted line. The step location is easily found by analyzing in which diatomic column the $Z$-contrast associated with $\operatorname{As}_{x} \mathrm{P}_{1-x}$ is no longer visible. This situation corresponds, for the contrast level of this image, to the existence of atomic columns of $\mathrm{P}$ atoms.

to AIP license or copyright; see http://apl.aip.org/apl/copyright.jsp 
explained by a purely strain-driven mechanism, without the need for introducing such a barrier.

Key evidence that nucleation occurs on the upper terrace comes from atomic resolution $Z$-contrast images, which show highest intensity from regions of the nanowire located on the upper terrace of the step. These regions correspond to the highest As composition, which is closest to the initial composition, with minimal intermixing during subsequent growth. They form in the region of maximum strain relaxation as predicted by the finite element calculations.

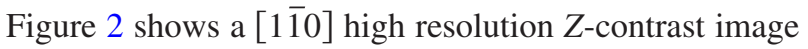
of an InAs nanowire of sample B. The nanowire was capped with InP after growth to prevent surface oxidation. This image was taken with a dedicated aberration-corrected STEM, a VG Microscope HB603U equipped with a Nion aberration corrector. The full width at half maximum of the electron probe is around $0.07 \mathrm{~nm}$. The high resolution aberrationcorrected Z-contrast imaging technique provides an incoherent image where the intensity on each probed atomic column, with the material properly oriented along a principal zone axis, strongly depends on $Z$, the atomic number of the atoms in the column. ${ }^{28-30}$

The studied material in our case is formed by a disordered substitutional $\operatorname{InAs}_{x} \mathrm{P}_{1-x}$ alloy with a chemical composition that changes from atomic column to atomic column throughout the studied nanowires. ${ }^{31}$ InAs-rich regions appear brighter in the image while a reduction of the intensity occurs as the InP content of the analyzed atomic columns increases. The shape and composition of the imaged nanowire are clearly identifiable in the image of Fig. 2 with atomic column spatial resolution. Contrast variations associated with changes in specimen thickness were minimized by selecting areas with negligible thickness variations. Local specimen thickness was monitored from measurements of the low loss region of the electron energy loss spectra (EELS) measured in an aberration-corrected VG Microscopes HB501UX equipped with a Gatan Enfina EEL spectrometer.

The interface between the nanowire and the substrate is highlighted with a dotted line in the high resolution $Z$-contrast image of Fig. 3. This image has been low pass filtered to remove high frequency noise without eliminating significant information from the original $Z$-contrast image. The chemical information contained in this $Z$-contrast image enables the location of a diatomic step at this interface below the imaged nanowire. A clear difference in contrast is observed between the In (brighter) and the As+P (dimmer) atomic columns. Note that the pure P columns are only barely detectable in the image of Fig. 2 and are not differentiated in the color rendering of the magnified image of Fig. 3. In this way, the presence of the faint column clearly delineates the outline of the nanowire and the presence of the interfacial step. Note that the step is seen with practically atomic abruptness, indicating that the step runs parallel to the beam direction for the entire sample thickness, i.e., this nanowire corresponds to the one circled in blue in Fig. 1. The existence of brighter intensities in the atomic columns of the nanowire located on the upper terrace of the diatomic step clearly confirms the presence of an As-rich region at this location. This locates the site of the original nucleation on the upper terrace and supports the strain-induced nucleation established in this letter from direct atomic force microscopy images and strain calculations.

In summary, from the direct imaging of nanowires, we have identified preferential sites for the nucleation of strained nanowires to lie on the upper terrace surface close to the step edge, where maximum stress relief occurs. We expect the same preferential sites to apply for negative strain also, where the upper terrace can again allow surface relaxation and provide preferential nucleation site.

This work was supported by the DOE Office of Basic Energy Sciences, Division of Materials Sciences and Engineering (MV and SJP), the SANDiE European Network of Excellence (Contract No. NMP4-CT-2004-500101), the Spanish MEC (TEC2005-05781-C03-01 y 02, NAN200409109-C04-01, and Consolider-Ingenio 2010 CSD200600019), the CAM (S 0505ESP 0200), and the Junta de Andalucia (PAI research groups TEP-120 and TIC-145; Project No. PAI05-TEP-00383).

${ }^{1}$ J. B. Hannon, V. B. Shenoy, and K. W. Schwarz, Science 313, 1266 (2006).

${ }^{2}$ P. W. Voorhees, Science 313, 1247 (2006).

${ }^{3}$ P. Venezuela, J. Tersoff, J. A. Floro, E. Chason, D. M. Follstaedt, F. Liu, and M. G. Lagally, Nature (London) 397, 678 (1999).

${ }^{4}$ S. Blunier, H. Zogg, C. Maissen, and A. N. Tiwari, Phys. Rev. Lett. 68, 3599 (1992).

${ }^{5}$ F. Liu, J. Tersoff, and M. G. Lagally, Phys. Rev. Lett. 80, 1268 (1998).

${ }^{6}$ V. M. Kaganer and K. H. Ploog, Phys. Rev. B 64, 205301 (2001).

${ }^{7}$ R. V. Kukta, A. Peralta, and D. Kouris, Phys. Rev. Lett. 88, 186102 (2002).

${ }^{8}$ J. Wollschlager, H. Pietsch, and A. Klust, Appl. Surf. Sci. 132, 29 (1998).

${ }^{9}$ P. Muller and A. Saul, Surf. Sci. Rep. 54, 157 (2004).

${ }^{10}$ L. G. Bai, J. Tersoff, and F. Liu, Phys. Rev. Lett. 92, 225503 (2004).

${ }^{11}$ D. E. Segall, C. Li, and G. Xu, Philos. Mag. 86, 5083 (2006).

${ }^{12}$ R. Leon, T. J. Senden, Y. Kim, C. Jagadish, and A. Clark, Phys. Rev. Lett. 78, 4942 (1997)

${ }^{13}$ A. C. Schindler, M. F. Gyure, G. D. Simms, D. D. Vvedensky, R. E. Caflisch, C. Connell, and E. Luo, Phys. Rev. B 67, 075316 (2003).

${ }^{14}$ A. P. Alivisatos, Science 271, 933 (1996).

${ }^{15}$ P. Michler, A. Kiraz, C. Becher, W. V. Schoenfeld, P. M. Petroff, L. Zhang, E. Hu, and A. Imamogùlu, Science 290, 2282 (2000).

${ }^{16}$ Z. Yuan, B. E. Kardynal, R. M. Stevenson, A. J. Shields, C. J. Lobo, K. Cooper, N. S. Beattie, D. A. Ritchie, and M. Pepper, Science 295, 102 (2002).

${ }^{17}$ M. Atatüre, J. Dreiser, A. Badolato, A. Högele, K. Karrai, and A. Imamoglu, Science 312, 551 (2006).

${ }^{18}$ J. Tersoff and R. M. Tromp, Phys. Rev. Lett. 70, 2782 (1993)

${ }^{19}$ D. Bimberg, M. Grundmann, and N. N. Ledentsov, Quantum Dot Heterostructures (Wiley, Chichester, UK, 1998), p. 37.

${ }^{20}$ J. Stangl, V. Holy, and G. Bauer, Rev. Mod. Phys. 76, 725 (2004).

${ }^{21}$ M. Grundmann, O. Stier, and D. Bimberg, Phys. Rev. B 52, 11969 (1995).

${ }^{22}$ J. M. García, L. González, M. U. González, J. P. Silveira, Y. González, and F. Briones, J. Cryst. Growth 227-228, 975 (2001).

${ }^{23}$ D. Fuster, B. Alen, L. Gonzalez, Y. Gonzalez, J. Martinez-Pastor, M. U. Gonzalez, and J. M. Garcia, Nanotechnology 18, 035604 (2007).

${ }^{24}$ D. Fuster, M. U. González, L. González, Y. González, T. Ben, A. Ponce, S. I. Molina, and J. Martínez-Pastor, Appl. Phys. Lett. 85, 1424 (2004).

${ }^{25}$ M. C. Xu, Y. Temko, T. Suzuki, and K. Jacobi, Surf. Sci. 589, 91 (2005).

${ }^{26}$ D. Leonard, K. Pond, and P. M. Petroff, Phys. Rev. B 50, 11687 (1994).

${ }^{27}$ M. Itoh and T. Ohno, Phys. Rev. B 62, 1889 (2000).

${ }^{28}$ P. E. Batson, N. Dellby, and O. L. Krivanek, Nature (London) 418, 617 (2002).

${ }^{29}$ P. D. Nellist, M. F. Chisholm, N. Dellby, O. L. Krivanek, M. F. Murfitt, Z. S. Szilagyi, A. R. Lupini, A. Borisevich, W. H. Sides, Jr., and S. J. Pennycook, Science 305, 1741 (2004).

${ }^{30}$ P. D. Nellist and S. J. Pennycook, Phys. Rev. Lett. 81, 4156 (1998).

${ }^{31}$ S. I. Molina, T. Ben, D. L. Sales, J. Pizarro, P. L. Galindo, M. Varela, S. J. Pennycook, D. Fuster, Y. Gonzalez, and L. Gonzalez, Nanotechnology 17, 5652 (2006). 